\title{
CTLA4Ig-mediated blockade of T-cell costimulation in patients with psoriasis vulgaris
}

\author{
Judith R. Abrams, ${ }^{1}$ Mark G. Lebwohl, ${ }^{2}$ Cynthia A. Guzzo, ${ }^{3}$ Brian V. Jegasothy, ${ }^{4}$ \\ Michael T. Goldfarb, ${ }^{5}$ Bernard S. Goffe, ${ }^{6}$ Alan Menter, ${ }^{7}$ Nicholas J. Lowe, ${ }^{8}$ \\ Gerald Krueger, ${ }^{9}$ Michael J. Brown, ${ }^{1}$ Russell S. Weiner, ${ }^{1}$ Martin J. Birkhofer, ${ }^{1}$ \\ Garvin L. Warner, ${ }^{10}$ Karen K. Berry, ${ }^{1}$ Peter S. Linsley, ${ }^{11}$ James G. Krueger, ${ }^{12}$ \\ Hans D. Ochs, ${ }^{13}$ Susan L. Kelley, ${ }^{1}$ and Sewon Kang ${ }^{5}$
}

\author{
${ }^{1}$ Bristol-Myers Squibb Pharmaceutical Research Institute, Wallingford, Connecticut 06492, USA \\ ${ }^{2}$ Department of Dermatology, Mount Sinai Medical Center, New York, New York 10029, USA \\ ${ }^{3}$ Department of Dermatology, University of Pennsylvania, Philadelphia, Pennsylvania 19104, USA \\ ${ }^{4}$ Department of Dermatology, University of Pittsburgh Medical Center, Montefiore University Hospital, \\ Pittsburgh, Pennsylvania 15213, USA \\ ${ }^{5}$ Department of Dermatology, University of Michigan, Ann Arbor, Michigan 48109, USA \\ ${ }^{6}$ Minor and James Medical Clinic, Seattle, Washington 98104-2138, USA \\ ${ }^{7}$ Baylor Psoriasis Research Center, Dallas, Texas 75246, USA \\ ${ }^{8}$ Clinical Research Specialists, Santa Monica, California 90404-2102, USA \\ ${ }^{9}$ Department of Dermatology, The University of Utah Health Science Center, Salt Lake City, Utah 84132-0001, USA \\ ${ }^{10}$ Genetics Institute, Andover, Massachusetts 01810, USA \\ ${ }^{11}$ Rosetta Inpharmatics, Kirkland, Washington 98034, USA \\ ${ }^{12}$ Laboratory for Investigative Dermatology, The Rockefeller University, New York, New York 10021-6399, USA \\ ${ }^{13}$ Division of Immunology, Infectious Diseases and Rheumatology, Department of Pediatrics, University of Washington School of \\ Medicine, Seattle, Washington 98195-6320, USA
}

Address correspondence to: Judith R. Abrams, Bristol-Myers Squibb Pharmaceutical Research Institute, 5 Research Parkway, Wallingford, Connecticut 06492, USA. Phone: (203) 677-7448; Fax: (203) 677-7690; E-mail: abramsj@bms.com

Portions of this work have appeared in abstract form (1997. J. Invest. Dermatol. 108:570a).

Received for publication November 20, 1998, and accepted in revised form March 15, 1999.

Engagement of the B7 family of molecules on antigen-presenting cells with their $\mathrm{T}$ cell-associated ligands, CD28 and CD152 (cytotoxic T lymphocyte-associated antigen-4 [CTLA-4]), provides a pivotal costimulatory signal in T-cell activation. We investigated the role of the CD28/CD152 pathway in psoriasis in a 26-week, phase I, open-label dose-escalation study. The importance of this pathway in the generation of humoral immune responses to T cell-dependent neoantigens, bacteriophage $\phi$ X174 and keyhole limpet hemocyanin, was also evaluated. Forty-three patients with stable psoriasis vulgaris received 4 infusions of the soluble chimeric protein CTLA4Ig (BMS-188667). Forty-six percent of all study patients achieved a $50 \%$ or greater sustained improvement in clinical disease activity, with progressively greater effects observed in the highest-dosing cohorts. Improvement in these patients was associated with quantitative reduction in epidermal hyperplasia, which correlated with quantitative reduction in skin-infiltrating $T$ cells. No markedly increased rate of intralesional $T$-cell apoptosis was identified, suggesting that the decreased number of lesional $\mathrm{T}$ cells was probably likely attributable to an inhibition of T-cell proliferation, T-cell recruitment, and/or apoptosis of antigen-specific T cells at extralesional sites. Altered antibody responses to $T$ cell-dependent neoantigens were observed, but immunologic tolerance to these antigens was not demonstrated. This study illustrates the importance of the $\mathrm{CD} 28 / \mathrm{CD} 152$ pathway in the pathogenesis of psoriasis and suggests a potential therapeutic use for this novel immunomodulatory approach in an array of $\mathrm{T}$ cell-mediated diseases.

J. Clin. Invest. 103:1243-1252 (1999).

\section{Introduction}

Psoriasis is a multifactorial disease of uncertain etiology that affects approximately $2 \%$ of the population (1). Psoriatic lesions are characterized by a clinical triad consisting of skin induration, scaling, and erythema. The histologic correlates of these clinical findings include inflammation, abnormal keratinocyte proliferation/terminal differentiation, and dermal angiogenesis. The inflammatory infiltrate, particularly pronounced at the dermal-epidermal junction, consists largely of activated
T cells and antigen-presenting cells (APCs) and precedes the development of epidermal hyperproliferation (2). Increased levels of inflammatory cytokines have been detected in lesional psoriatic epidermis, which may result in the potentiation of T-cell activation (3) as well as hyperproliferation and accelerated differentiation of keratinocytes $(4,5)$. These and other data derived from $\mathrm{T}$ cell-based therapeutics (6-8) suggest that activated $\mathrm{T}$ cells play an important role in triggering and perpetuating the disease. 
Day 1
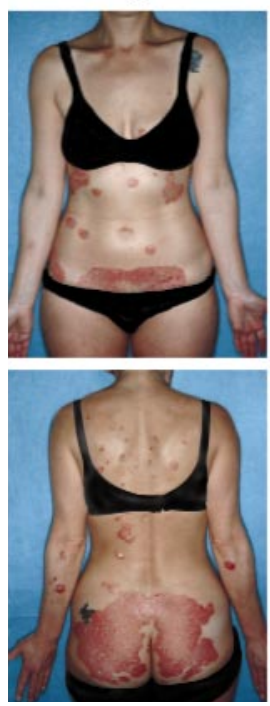

Day 36
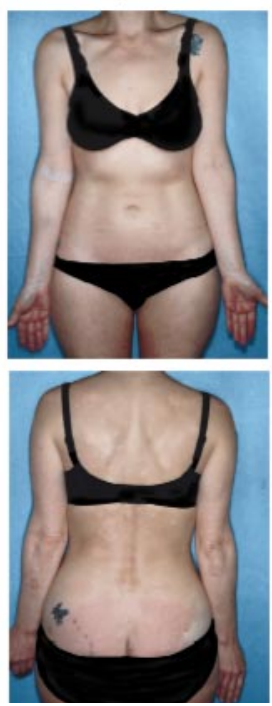

Day 71
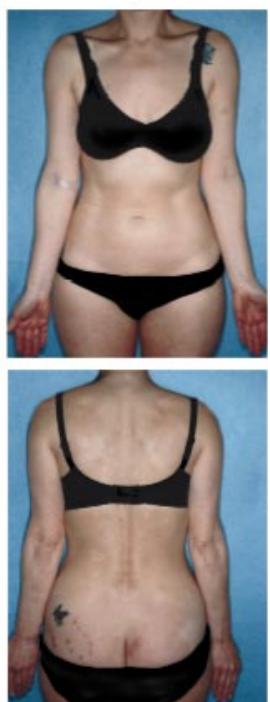

Figure 1

Clinical findings representative of a $50 \%$ or greater improvement from baseline Physician's Global Assessment observed in 19 of 41 study patients. Serial photographs are obtained at baseline (left panels), day 36 (middle panels), and day 71 (right panels) in a patient accrued to the CTLA4lg $50 \mathrm{mg} / \mathrm{kg}$ dose level. A maximal improvement of $90 \%$, compared with baseline, was reported for this patient.

The B7 family of molecules on APCs regulate T-cell activation by delivering antigen-independent stimulatory signals through CD28 and inhibitory signals through CD152 (cytotoxic T lymphocyte-associated antigen-4 [CTLA-4]) $(9,10)$. CTLA4Ig (BMS-188667) is a soluble chimeric protein consisting of the extracellular domain of human $\mathrm{CD} 152$ and a fragment (hinge, $\mathrm{CH} 2$, and $\mathrm{CH} 3$ domains) of the Fc portion of human IgG1 (11). CTLA4Ig binds to B71 (CD80) and B7-2 (CD86) molecules on APCs and thereby blocks the $\mathrm{CD} 28$-mediated costimulatory signal for $\mathrm{T}$ cell activation. Biologic activity of CTLA4Ig has been demonstrated in a variety of animal models of transplantation (12-16) and autoimmunity (17-20). The biologic effects of CTLA4Ig in some transplantation models have been reported to persist well after the clearance of all detectable drug from the circulation. Occasionally, donorspecific tolerance has been observed (13-15). In some animal models of autoimmunity, CTLA4Ig not only prevents the induction of an autoimmune process but also suppresses disease activity late in the course of an established autoimmune response (18-20).

We evaluated the role of ongoing T-cell costimulation in the development and perpetuation of psoriatic plaques. Prior in vitro experiments have shown that CTLA4Ig inhibits, in a dose-dependent fashion, the capacity of B7 molecules present on epidermal Langerhans' cells and dermal dendritic cells to serve as costimulatory molecules for the proliferation of T cells in a primary immune response (21-23). The importance of the CD28/CD152 pathway in a chronic cutaneous $T$ cell-mediated disease such as psoriasis was previously unknown. We also assessed the ability of CTLA4Ig to alter a humoral immune response to $2 \mathrm{~T}$-dependent neoantigens, bacteriophage $\phi X 174$ and keyhole limpet hemocyanin (KLH). The findings in this phase I clinical study suggest that the blockade of T-cell costimulatory signals mediated by the B7 family of molecules may be a potent strategy of immune modulation in psoriasis and other $\mathrm{T}$ cell-mediated diseases.

\section{Methods}

Study design and patient characteristics. This phase I, multicenter, open-label dose-escalation study was approved by the ethics committee at each participating center. Patients providing informed consent for use of the investigational agents were enrolled in this study if they had a history of stable psoriasis vulgaris of at least 6 months' duration (involving 10-49\% of total body surface area) and had failed at least 1 prior anti-psoriatic therapy. No evidence of active bacterial or viral infections was present at the time of enrollment. Prior to enrollment, retinoids were discontinued for at least 2 years; investigational drugs, methotrexate, cyclosporine, and systemic corticosteroids were discontinued for at least 16 weeks; phototherapy and photochemotherapy were not administered for at least 4 weeks; topical treatments other than emollients were not administered for 2 or more weeks. Of the 43 patients who were treated in the study, 35 (81\%) were men and 8 (19\%) were women. Patients' ages ranged from 23 to 57 years (median age: 39 years). The median duration of psoriasis was 16.4 years, with an overall range of $0.7-43.0$ years. The median body surface area involvement at day 1 was $25 \%$ (range: $11-55 \%$ ). Previously failed therapies included topical corticosteroids $(n=37)$, phototherapy $(n$ $=31)$, calcipotriol $(n=19)$, systemic corticosteroids $(n=15)$, coal $\operatorname{tar}(n=12)$, methotrexate $(n=8)$, etretinate $(n=6)$, and cyclosporine $(n=6)$. Twenty-three psoriatic patients matched to this study population for age and overall disease severity served as a parallel control group. Baseline characteristics were similar in all groups.

CTLA4Ig administration and evaluation of patients. CTLA4Ig (BMS-188667) was administered as a 1-hour intravenous infusion on day 1 (week 1), day 3 (week 1), day 16 (week 3), and day 29 (week 5). The starting dose of CTLA4Ig in this open-label

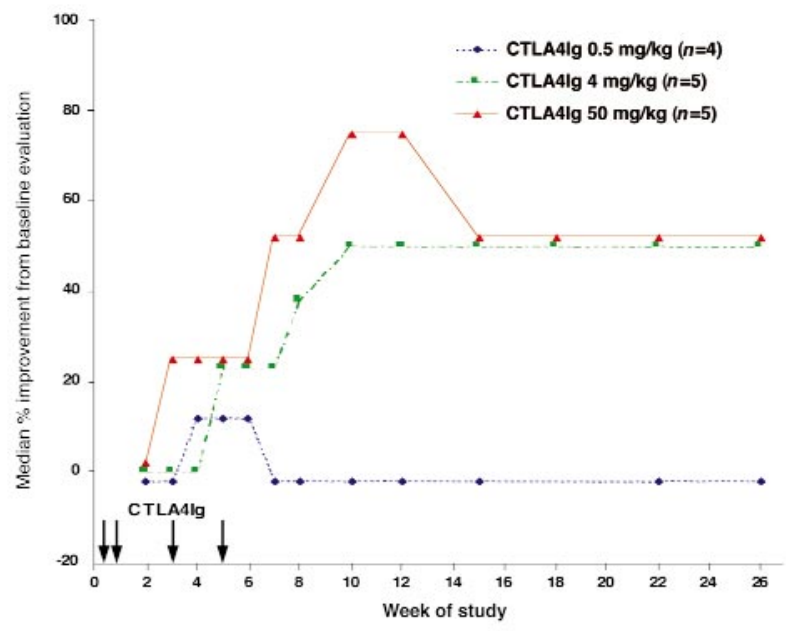

Figure 2

Median Physician's Global Assessment of disease activity in the CTLA4/g $0.5 \mathrm{mg} / \mathrm{kg}, 4 \mathrm{mg} / \mathrm{kg}$, and $50 \mathrm{mg} / \mathrm{kg}$ dose groups during the study. A significant dose response across the 8 CTLA4Ig treatment groups $(P<0.05)$ was detected at study weeks $3,4,10,12$, and 15 ; patient numbers were too limited in some dose groups at study weeks 18-26 to allow statistical comparison. 
dose-escalation study was $0.5 \mathrm{mg} / \mathrm{kg}$. Four to 6 patients were accrued to each of 8 dose levels: $0.5 \mathrm{mg} / \mathrm{kg}, 1 \mathrm{mg} / \mathrm{kg}, 2 \mathrm{mg} / \mathrm{kg}, 4$ $\mathrm{mg} / \mathrm{kg}, 8 \mathrm{mg} / \mathrm{kg}, 16 \mathrm{mg} / \mathrm{kg}, 25 \mathrm{mg} / \mathrm{kg}$, and $50 \mathrm{mg} / \mathrm{kg}$. There was no intrapatient dose escalation. Patients were monitored for a 4-hour period following each infusion, at weekly intervals during the first 8 weeks of study, and then at biweekly-to-monthly intervals through a follow-up of 176 days' median duration (week 26). Safety (hematology and biochemistry, including serum protein electrophoresis, immunophenotyping, and urine studies), immunogenicity, pharmacokinetic, and biologic activity assessments (antibody titers; Physician's Global Assessment) were performed at each of these scheduled visits. The Physician's Global Assessment, an evaluation of the extent/worsening of the patient's condition relative to pretreatment, was performed in the traditional manner employing a 7-point scale, with the degree of improvement compared with baseline evaluation denoted in the following manner: $0=100 \%$, completely clear; 1 $=90 \% ; 2=75 \% ; 3=50 \% ; 4=25 \% ; 5=0 \% ; 6=$ deterioration (24).

Bacteriophage $\phi X 174$ and KLH-ImmuneActivator immunization protocol. Bacteriophage $\phi X 174$ (provided by H.D. Ochs, University of Washington, Seattle, Washington, USA) was administered as an intravenous bolus at a dose of $0.02 \mathrm{~mL} / \mathrm{kg}$ body weight $\left(2 \times 10^{9}\right.$ plaque-forming units $/ \mathrm{kg}$ ) on days 1 (week 1$), 29$ (week 5), 71 (week 11), and 148 (week 22). KLH-ImmuneActivator (INTRACEL Corp., Rockville, Maryland, USA) was administered at a dose of $1.0 \mathrm{mg}$ intradermally on day -14 (week -2) and day 29 (week 4) to patients accrued to the CTLA4Ig dose levels above $0.5 \mathrm{mg} / \mathrm{kg}$ and to 19 of 23 patients accrued to the control cohort; all other study patients received a dose of $0.1 \mathrm{mg}$ intradermally at the appointed times. Patient sera were collected pre and 1,2 , and 4 weeks after each administration of bacteriophage $\phi X 174$ or KLH-ImmuneActivator for analysis of antibody titers.

CTLA4Ig serum level determination and pharmacokinetics. CTLA4Ig serum concentrations were determined using a validated ELISA with a lower level of CTLA4Ig quantitation of 1 $\mathrm{ng} / \mathrm{mL}(25)$. Values were determined by a standard curve employing known quantities of CTLA4Ig in 10\% human serum. Within the dose range of $0.5-50 \mathrm{mg} / \mathrm{kg}$, CTLA4Ig exhibited linear pharmacokinetics, with little apparent variability between patients. The CTLA4Ig mean peak serum concentrations $(\mathrm{Cmax} \pm \mathrm{SD})$ following administration of the fourth dose were $17.0 \pm 4.6 \mu \mathrm{g} / \mathrm{mL}$ and $2,201 \pm 578 \mu \mathrm{g} / \mathrm{mL}$ at the 0.5 and 50 $\mathrm{mg} / \mathrm{kg}$ dose levels, respectively. The mean serum elimination half-life (T-HALF) of CTLA4Ig was 14.7 days; there was no apparent change in the T-HALF with increasing doses of CTLA4Ig. The rate of elimination remained constant following dosing, reflecting the lack of anti-CTLA4Ig antibody generation following infusion of CTLA4Ig.

Anti-CTLA4Ig antibody determination. Sera were collected from study patients to detect anti-CTLA4Ig antibodies at baseline and then at weekly-to-monthly intervals through the final study day (day 176). An ELISA was employed in the analyses that utilized CTLA4Ig as a capture reagent (plates coated with $2 \mu \mathrm{g} / \mathrm{mL}$ CTLA4Ig). Specific antibody binding was detected using a mixture of commercially available, alkaline phosphatase-conjugated, goat anti-human $\kappa$ and $\lambda$ light chain antibodies (Southern Biotechnology Associates Inc., Birmingham, Alabama, USA).
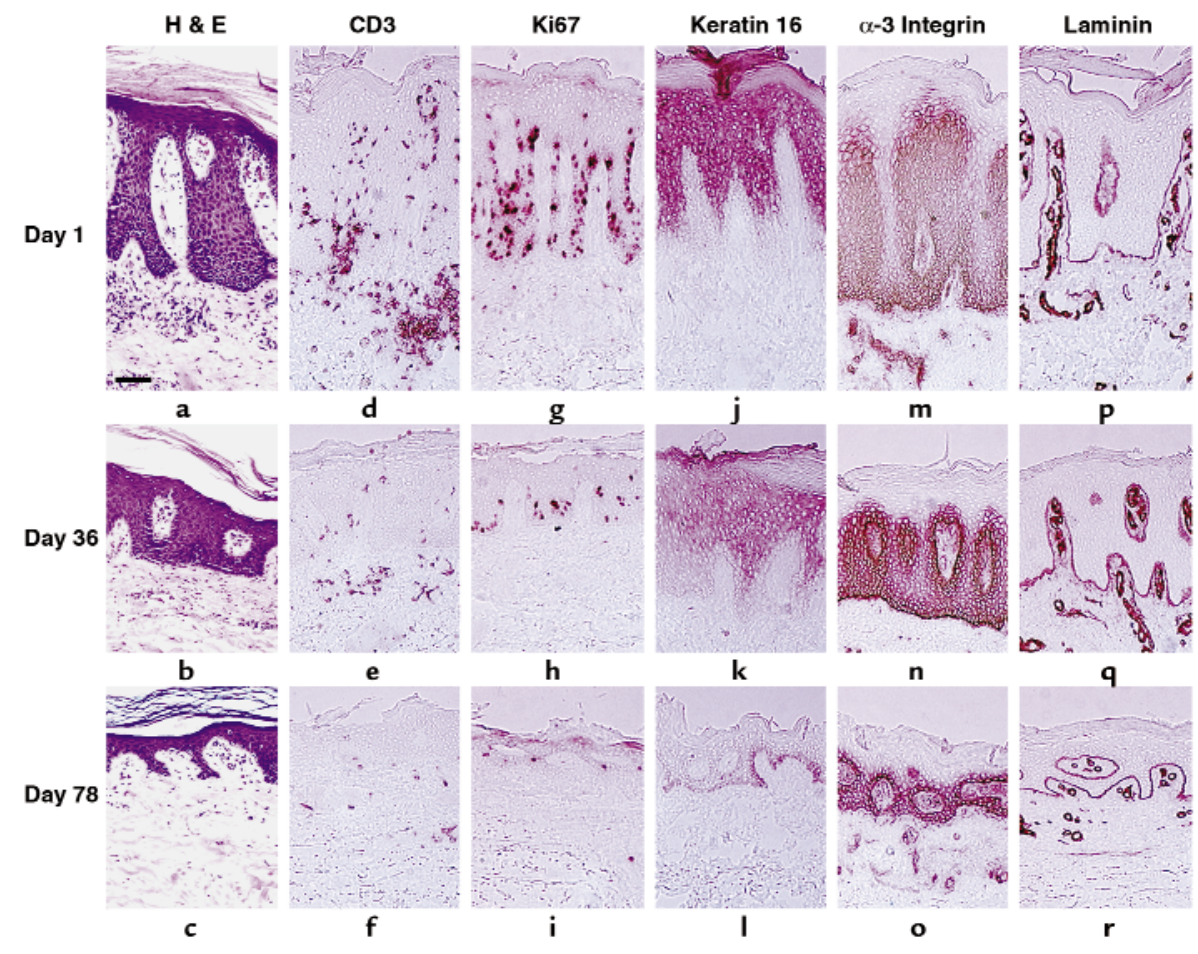

g
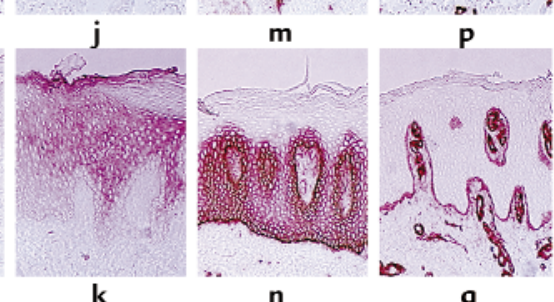

h

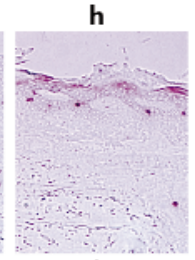

k

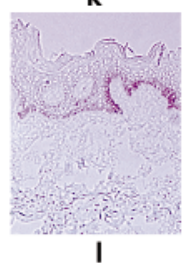

n

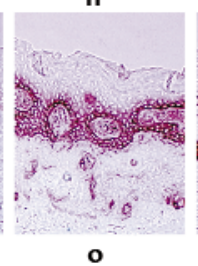

9

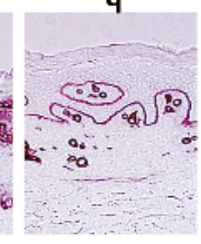

Figure 3

Reversal of molecular markers of epidermal and vascular pathology following administration of CTLA4lg. Representative immunohistologic findings in the 19 patients demonstrating a 50\% or greater improvement in global clinical parameters following administration of CTLA4lg. Serial biopsies at day 1 (upper row), day 36 (middle row), and day 78 (lower row) obtained from the perimeter of a single representative lesion in a patient accrued to the CTLA4Ig $25 \mathrm{mg} / \mathrm{kg}$ dose level. Hematoxylin and eosin-stained sections (labeled H \& E in a-c) demonstrate progressive epidermal thinning, diminution in the inflammatory cellular infiltrate, and normalization of keratinocyte maturation on or prior to day 36 . Scale bar in a: $100 \mu \mathrm{m}$. T cells and proliferating cells present in the psoriatic lesions were detected by immunostaining with mAb's to CD3 (d-f) and Ki67 nuclear protein ( $\mathbf{g}-\mathbf{i}$ ), respectively. A progressive decrease in the number of positively staining cells was evident in the serial biopsies. Expression of keratin $16(j-I)$ and $\alpha-3$ integrin $(\mathbf{m}-\mathbf{o})$ in lesional biopsies was reduced following administration of CTLA4lg. Immunostaining with mAb's to laminin (p-r), present in the basement membrane of blood vessels, illustrates the serial decrease in the ectasia of the lesional vessels. 
Table 1

Biologic end points by dose level on study

\begin{tabular}{|c|c|c|c|c|c|c|c|}
\hline \multirow[t]{2}{*}{$\begin{array}{l}\text { CTLA4Ig } \\
\text { dose }\end{array}$} & \multirow[t]{2}{*}{$\begin{array}{l}\quad \geq 50 \% \text { improvement in } \\
\text { Physician's Global Assessment }{ }^{\mathrm{A}}\end{array}$} & \multirow{2}{*}{$\begin{array}{c}\text { Mean \% reduction in } \\
\text { epidermal T cells } \\
\text { Day } 78\end{array}$} & \multicolumn{2}{|c|}{$\begin{array}{l}\text { Mean \% reduction } \\
\text { in epidermal thickness }{ }^{\mathrm{B}}\end{array}$} & \multicolumn{3}{|c|}{ Antibody titers } \\
\hline & & & Day 36 & Day 78 & $\phi \times 1741^{\circ} \mathrm{C}$ & $\phi \times 1742^{\circ \mathrm{D}}$ & $\mathrm{KLH} 2^{\circ \mathrm{D}}$ \\
\hline $\mathrm{mg} / \mathrm{kg}$ & No. of subjects & $\%$ & $\%$ & $\%$ & No. of subjects & below mean & trol group \\
\hline 0 & $1 / 23$ & - & - & - & - & - & - \\
\hline 0.5 & $0 / 4$ & 51 & 2 & -18 & $2 / 4$ & $2 / 4$ & - \\
\hline 1 & $1 / 5$ & 75 & 15 & 53 & $2 / 5$ & $2 / 5$ & $1 / 5$ \\
\hline 2 & $1 / 5$ & 82 & 30 & 52 & $3 / 5$ & $2 / 5$ & $3 / 5$ \\
\hline 4 & $3 / 5$ & 71 & 40 & 57 & $3 / 5$ & $2 / 4$ & $1 / 4$ \\
\hline 8 & $3 / 6$ & 56 & 44 & 16 & $6 / 6$ & $6 / 6$ & $3 / 6$ \\
\hline 16 & $2 / 5$ & $67^{\mathrm{E}}$ & 32 & $57^{\mathrm{E}}$ & $5 / 6$ & $6 / 6$ & $4 / 6$ \\
\hline 25 & $5 / 6$ & $82^{\mathrm{F}}$ & $45^{\mathrm{F}}$ & $51^{\mathrm{F}}$ & $6 / 6$ & $6 / 6$ & $3 / 6$ \\
\hline 50 & $4 / 5$ & $93^{\mathrm{F}}$ & $47^{F}$ & $61^{\mathrm{F}}$ & $4 / 6$ & $3 / 5$ & $1 / 5$ \\
\hline
\end{tabular}

T cell-mediated and T-dependent humoral biologic end points were studied following administration of CTLA4lg at unit doses ranging between $0.5 \mathrm{and} 50 \mathrm{mg} / \mathrm{kg}$. A parallel control group $(0 \mathrm{mg} / \mathrm{kg})$ received all clinical assessments and immunizations but were not administered CTLA4lg infusions. APhysician's Global Assessment is an evaluation of the extent of improvement or worsening of the patient's condition, relative to pretreatment. A response was defined as a $50 \%$ or greater improvement observed on 2 or more consecutive visits over the study period. Two patients who received CTLA4lg were not evaluable for clinical response: one patient accrued to the $16 \mathrm{mg} / \mathrm{kg}$ dose level received concomitant prednisone $(5 \mathrm{mg} /$ day $)$ and an incorrect dose $(2 \mathrm{mg} / \mathrm{kg}$ ) of CTLA4lg on day 3; a patient enrolled in the $50 \mathrm{mg} / \mathrm{kg}$ dose level was administered intravenous steroids on study day 32 for an exacerbation of asthma. ${ }^{B}$ Mean percent change was calculated by computing the mean $\log _{10}$ ratio of the day 36 or day 78 value to the baseline (day 1 ) value. The mean $\log$ ratio was then transformed to the percent change scale according to the following: mean percent change $=100 \times$ $\left(10^{M L R}-1\right)$, where $M L R=$ mean $\log$ ratio. ${ }^{C} A$ patient was defined as a responder if the titers at 2 weeks and/or 4 weeks after primary bacteriophage $\phi X 174$ immunization were suppressed to values $2 \mathrm{SDs}$ or greater below the geometric mean of a parallel control population (denoted as CTLA4lg dose of $0 \mathrm{mg} / \mathrm{kg}$ ). ${ }^{D} \mathrm{~A}$ patient was defined as a responder if at least 2 antibody titers obtained following secondary immunization and prior to the tertiary immunization were suppressed to values 2 SDs or greater below the geometric mean of a parallel control population. Patients accrued to the $0.5 \mathrm{mg} / \mathrm{kg}$ dose level received KLH at a dose of $0.1 \mathrm{mg}$ intradermally at day -14; a nominal humoral antibody response to KLH was observed, and summary statistics were not tabulated. Patients accrued to all subsequent dose levels were administered KLH at a set dose of $1.0 \mathrm{mg}$ intradermally at days -14 and 29 . $E P<0.05$, and $F P<0.01$ based on two-sided $t$ test for no change from baseline.

Serum samples were serially diluted 2 -fold; the end-point titer was the reciprocal of the greatest dilution of sera that resulted in an $\mathrm{A}_{450}$ at least 5 times greater than the plate background. Seroconversion was defined as a minimum 4 -fold increase in end-point titer at any study day relative to baseline examination.

Anti-bacteriophage $\phi X 174$ antibody and anti-KLH antibody measurements. Anti-bacteriophage antibody was assayed as neutralizing activity and expressed as the $\mathrm{K}$ value $(\mathrm{Kv})$, the rate of phage inactivation over time (26). The titer of anti-KLH antibodies was measured by ELISA (27). Values were determined by a standard curve employing sera containing a known quantity of anti-KLH antibody (a gift from C.O. Elson, University of Alabama-Birmingham, Birmingham, Alabama, USA).

Peripheral blood leukocyte immunophenotyping. Blood samples for immunophenotyping were obtained prior to administration of CTLA4Ig (days -14 and 1) and at study days 8, 22, 36, 64, 99, and 148. Single cell suspensions for flow cytometry were incubated with combinations of the following mouse anti-human, fluorochrome-conjugated mAb's at saturation for 15 minutes at room temperature and fixed in PBS containing 1\% paraformaldehyde: (a) FITC-conjugated anti-CD8 [SK1]; antiCD25 [2A3]; anti-CD28 [L293]; anti-CD45 [2D1]; antiCD45RA [L48]; anti-TCR- $\alpha / \beta$ [WT31] (Becton Dickinson Immunocytometry Systems, San Jose, California, USA); antiCD40 [EA5]; anti-CD86 [BU63]; anti-CD154 [24.31] (Ancell Corp., Bayport, Minnesota, USA); anti-CD54 [15.2] (BIODESIGN International, Kennebunk, Maine, USA); anti-CD80 [BB1] (PharMingen, San Diego, California, USA); (b) phycoerythrin-conjugated (PE-conjugated) anti-CD4 [SK3]; antiCD14 [MOP9]; anti-CD19 [4G7]; CD45RO [UCHL-1]; antiCD56 [MY31]; anti-CD80 [L307.4]; TCR- $\gamma / \delta$ [11F2]; HLA-DR [L243] (Becton Dickinson Immunocytometry Systems); antiCD86 [FUN-1] (PharMingen); (c) peridinin chlorophyll protein-conjugated (PerCP-conjugated) anti-CD3 [SK7]; anti-CD4 [SK3]; anti-CD8 [SK1] (Becton Dickinson Immuno- cytometry Systems); (d) Tri-Color-conjugated (TC-conjugated) anti-CD14 [TUK4]; anti-CD19 [SJ25.C1] (Caltag Laboratories Inc., Burlingame, California, USA). The controls for nonspecific Ig binding were mouse IgG1 directly conjugated to FITC, PE, or PerCP (Becton Dickinson Immunocytometry Systems); mouse IgG2a directly conjugated to PE or TC (Caltag Laboratories Inc.); and mouse IgM-FITC (PharMingen). Ten thousand lymphocyte-gated or 1,000 monocyte-gated events were collected for triple staining on a Cytoron Absolute flow cytometer and data were analyzed using Immunocount software (both from Ortho Diagnostic Systems Inc., Raritan, New Jersey, USA).

Pathologic analysis. Five serial samples for histologic analysis were obtained with a 6 - $\mathrm{mm}$ punch biopsy from a single representative lesion before administration of CTLA4Ig and at study days 8, 16, 36, and 78. Each specimen was split for routine histopathology and for histochemical analysis on cryostat-cut $6-\mu \mathrm{m}$ sections. Immunohistochemical staining procedures were performed as previously described $(7,28)$. Serial sections from selected patients were also processed using a terminal deoxynucleotidyl transferase-mediated dUTP nick endlabeling (TUNEL) assay (29). Terminal deoxynucleotidyl transferase (TdT; Pharmacia Biotech AB, Uppsala, Sweden) and biotin-16-dUTP (Boehringer Mannheim Biochemicals Inc., Indianapolis, Indiana, USA) were used in these reactions. As positive controls, sections treated with DNase (Sigma Chemical Co., St. Louis, Missouri, USA) at $1 \mathrm{U} / \mathrm{mL}$ and biopsy specimens obtained from patients treated with ultraviolet B light were included with each experiment.

Statistical analysis. Data are summarized as frequencies for categorical data or means and confidence intervals for continuous data. Confidence intervals were calculated based on the $t$ distribution. For transformed variables, the summaries were computed on the transformed data and then reported on the original scale. Comparisons of on-study values with baseline were made with a paired $t$ test. Longitudinal com- 
parisons of Physician's Global Assessment among the dose groups were done at each time point using an exact test for a linear-by-linear association, with dose level and percent improvement used as category weights (30). No adjustments were made for multiple comparisons.

\section{Results}

Clinical response to CTLA4Ig. Nineteen (46\%) of the 41 patients evaluable for clinical response achieved a $50 \%$ or greater improvement in their Physician's Global Assessment of disease activity, compared with baseline psoriasis evaluation (Table 1 and Figure 1). In contrast, only 1 (4\%) of 23 patients in a parallel control group of psoriatic patients spontaneously exhibited a $50 \%$ or greater improvement in the Physician's Global Assessment. When comparisons are made across the 8 CTLA4Ig treatment groups, a significant dose response was demonstrated $(P<0.05)$ at study weeks $3,4,10,12$, and 15 ; patient numbers were too limited in some dose groups at study weeks 18-26 to allow statistical comparison. A 50\% or greater improvement in global clinical parameters of psoriasis was observed in 9 of 11 patients accrued to the top 2 CTLA4Ig dosing cohorts and in 1 of 9 patients enrolled in the 2 lowest dosing levels. Sustained clinical improvement was observed in some cases for at least 147 days following administration of the final dose of CTLA4Ig (Figure 2). In general, concordant responses were observed in dosing cohorts across the various biologic response endpoints.

CTLA4Ig was well tolerated in this study. There were no reports of clinically significant adverse sequelae of immunosuppression, such as opportunistic infections or malignancy. Serial immunophenotyping studies revealed no detectable cellular depletion of B7-bearing target cell populations or alterations in lymphocyte subset distribution. Suppression of total serum immunoglobulins and increases in anti-CTLA4Ig antibody titers were not observed. The most common adverse events reported throughout the period of obser- vation (median duration: 176 days) were uncomplicated upper respiratory tract infection (16\%) and transient headache (16\%). Five of the 7 reported cases of upper respiratory tract infection occurred at CTLA4Ig unit doses of $2 \mathrm{mg} / \mathrm{kg}$ or less. No patients discontinued treatment because of adverse events. One patient accrued to the 50 $\mathrm{mg} / \mathrm{kg}$ dose level required hospitalization for exacerbation of preexisting asthma 3 days following the fourth (day 29) administration of CTLA4Ig and the second dose of KLH and bacteriophage $\phi X 174$.

Reversal of keratinocyte maturational abnormalities and vascular pathology. Histologic changes in psoriatic lesions were studied using immunohistochemistry on cryostatprepared skin specimens obtained serially from a single representative lesion prior to CTLA4Ig administration (day 1 ) and at days 8, 16, 36, and 78 following the initial infusion. Clinical improvement was associated with reversal of disease-defining pathology in serial biopsies. All study patients who attained a $50 \%$ or greater improvement in global clinical parameters of psoriasis displayed a normalization of keratinocyte maturation/proliferation analogous to those changes illustrated in Figure 3. Reformation of the granular cell layer, absence of retained keratinocyte nuclei in the stratum corneum (parakeratosis), and a reversion of the thickened stratum corneum (hyperkeratosis) to a "basketweave" configuration are all characteristic of the normalization of the keratinocyte terminal differentiation process (Figure 3, a-c). Cellular infiltration with $\mathrm{CD}^{+}(\mathrm{T})$ cells, most marked at the dermal-epidermal junction, was serially decreased following day 1 dosing (Figure 3, d-f). The expanded pool of cycling keratinocytes became restricted to the basal layer, as seen in normal skin (Figure 3, g-i). Keratins (Figure 3, j-1) and integrins (Figure 3, m-o) expressed in the basal and suprabasal layers of the hyperplastic psoriatic epidermis (31) were confined to the basal layer of keratinocytes in the subset of patients demonstrating a $50 \%$ or greater

Table 2

Bacteriophage $\phi \times 174$ antibody response at 2 weeks after primary and secondary immunization with bacteriophage $\phi \times 174$

\begin{tabular}{|c|c|c|c|c|c|c|c|c|}
\hline \multirow[t]{2}{*}{ CTLA4II dose } & \multicolumn{3}{|c|}{ Two weeks post primary immunization } & \multicolumn{5}{|c|}{ Two weeks post secondary immunization } \\
\hline & $n$ & Mean titer ${ }^{A}$ & $\begin{array}{l}95 \% \text { confidence } \\
\text { interval }\end{array}$ & $n$ & Mean titer ${ }^{A}$ & $\begin{array}{l}95 \% \text { confidence } \\
\text { interval }\end{array}$ & Mean $\% \lg G^{B}$ & $\begin{array}{c}95 \% \text { confidenc } \\
\text { interval }\end{array}$ \\
\hline $\mathrm{mg} / \mathrm{kg}$ & & Kv & & & $\mathrm{Kv}$ & & $\%$ & \\
\hline 0 & 23 & 125 & $(69,227)$ & 21 & 410 & $(259,569)$ & 27 & $(20,34)$ \\
\hline 0.5 & 4 & 11 & $(2,82)$ & 3 & 33 & $(0,3,717)$ & 7 & $(0,31)$ \\
\hline 1 & 5 & 16 & $(6,46)$ & 5 & 168 & $(48,591)$ & 4 & $(1,10)$ \\
\hline 2 & 5 & 13 & $(2,82)$ & 5 & 95 & $(14,647)$ & 2 & $(0,7)$ \\
\hline 4 & 5 & 11 & $(3,40)$ & 4 & 69 & $(10,495)$ & 5 & $(0,16)$ \\
\hline 8 & 6 & 6 & $(3,11)$ & 6 & 15 & $(3,79)$ & 1 & $(0,2)$ \\
\hline 16 & 6 & 3 & $(1,13)$ & 6 & 8 & $(3,24)$ & 0 & $(0,2)$ \\
\hline 25 & 6 & 4 & $(2,9)$ & 6 & 18 & $(4,87)$ & 1 & $(0,2)$ \\
\hline 50 & 6 & 9 & $(2,49)$ & 5 & 28 & $(1,629)$ & 2 & $(0,6)$ \\
\hline
\end{tabular}

The ability of CTLA4Ig to alter a T-dependent humoral immune response to bacteriophage $\phi X 174$ was assessed in all study patients. A parallel control group ( 0 mg/kg) was administered bacteriophage $\phi X 174$ under the identical immunization schedule but did not receive CTLA4Ig infusions. The primary immunization with bacteriophage $\phi X 174$ was concurrent with the day 1 infusion with CTLA4lg; the secondary immunization was concurrent with the fourth CTLA4Ig infusion (day 29). Results at 2 weeks following primary and secondary immunization are shown. Antibody titers are expressed as the rate of phage inactivation, or K value (Kv), as described in Methods. ${ }^{A} T h e$ geometric mean titer of anti-bacteriophage antibody was derived from the arithmetic mean of the $\log _{10}$ titer, with subsequent transformation back to the original titer scale. ${ }^{B}$ Individual isotypes of anti-phage antibody in sera were measured by the ability of in vitro treatment with 2-mercaptoethanol to abolish activity of the IgM, but not the IgG isotype antibody. The mean and confidence interval were calculated using the arcsin transformation $\left[y=\sin ^{-1}(\sqrt{ } \mathrm{X})\right]$ and then transformed back to the percent scale for reporting. 
Table 3

Mean total anti-KLH antibody response following secondary immunization with $\mathrm{KLH}$

\begin{tabular}{|c|c|c|c|c|c|c|}
\hline \multirow[t]{2}{*}{ CTLA4lg dose $\mathrm{A}^{\mathrm{A}}$} & \multicolumn{3}{|c|}{ Two weeks after secondary immunization } & \multicolumn{3}{|c|}{ Four weeks after secondary immunization } \\
\hline & $n$ & $\begin{array}{l}\text { Mean anti-KLH } \\
\text { antibody }{ }^{B}\end{array}$ & $\begin{array}{c}95 \% \text { confidence } \\
\text { interval }\end{array}$ & $n$ & $\begin{array}{c}\text { Mean anti-KLH } \\
\text { antibody }^{\mathrm{B}}\end{array}$ & $\begin{array}{l}95 \% \text { confidence } \\
\text { interval }\end{array}$ \\
\hline $\mathrm{mg} / \mathrm{kg}$ & & $\mu \mathrm{g} / \mathrm{ml}$ & & & $\mu \mathrm{g} / \mathrm{ml}$ & \\
\hline 0 & 19 & 747 & $(515,1,085)$ & 19 & 628 & $(457,863)$ \\
\hline 1 & 5 & 506 & $(125,2,047)$ & 5 & 370 & $(100,1,375)$ \\
\hline 2 & 5 & 184 & $(42,808)$ & 5 & 108 & $(26,453)$ \\
\hline 4 & 4 & 230 & $(59,894)$ & 4 & 228 & $(54,961)$ \\
\hline 8 & 6 & 108 & $(27,428)$ & 6 & 81 & $(18,354)$ \\
\hline 16 & 6 & 136 & $(69,270)$ & 5 & 78 & $(61,102)$ \\
\hline 25 & 6 & 105 & $(16,683)$ & 6 & 84 & $(13,549)$ \\
\hline 50 & 5 & 982 & $(74,13,000)$ & 5 & 813 & $(109,6,071)$ \\
\hline
\end{tabular}

Alteration in the anti-KLH antibody response was assessed in all study patients following administration of CTLA4lg. A parallel control group (0 mg/kg) was administered $\mathrm{KLH}$ under the identical immunization schedule but did not receive CTLA4Ig infusions. The primary KLH immunization occurred 2 weeks prior to the first infusion with CTLA4lg; (day 1 ); the secondary immunization was concurrent with the fourth infusion with CTLA4Ig (day 29). ${ }^{A}$ Four of 23 subjects accrued to the control cohort (0 mg/kg) and all subjects accrued to the CTLA4lg $0.5 \mathrm{mg} / \mathrm{kg}$ dose level, demonstrated negligible primary anti-KLH antibody titers following immunization with 0.1 mg KLH intradermally; summary statistics were not tabulated for the $0.5 \mathrm{mg} / \mathrm{kg}$ dosing cohort. The remaining 19 patients accrued to the control cohort, and patients accrued to the $1 \mathrm{mg} / \mathrm{kg}$ and all subsequent dose levels were administered KLH at a set dose of $1.0 \mathrm{mg}$ intradermally at days -14 and 29 . Results at 2 and 4 weeks following the secondary KLH immunization are shown. The titer of anti-KLH antibodies was measured by ELISA, and the values were determined by a standard curve employing sera containing a known quantity of KLH antibody. ${ }^{\mathrm{B} T h e}$ geometric mean titer was derived from the arithmetic mean of the $\log _{10}$ titer, with subsequent transformation back to the original titer scale.

clinical improvement following CTLA4Ig administration. A decreased dermal angiogenic tissue reaction was also noted in this subset of patients (Figure 3, p-r).

Quantitative reduction in epidermal byperplasia correlates with reduction in measures of $T$-cell infiltration in psoriatic lesions. Computer-assisted image analysis was employed to quantify changes in epidermal thickness, proliferating (Ki67 nuclear antigen-positive) epidermal cells (32), and $\mathrm{CD}^{+}(\mathrm{T})$ cell infiltration in serial biopsies from a sole representative lesion in each patient. Alterations in these objective histologic measures following administration of CTLA4Ig were concordant with the clinical responses noted above (Table 1). Some degree of improvement in all quantitative histologic measures was noted at all dose levels. The most consistent and statistically significant reductions, however, were observed in the 11 patients accrued to the top 2 dosing cohorts. Quantitative data derived from all patients accrued to the CTLA4Ig 25 $\mathrm{mg} / \mathrm{kg}$ dosing cohort, reflective of the magnitude, consistency, and cadence of the response observed at the top 2 dose levels, are illustrated in Figure 4.

Reductions in epidermal thickness (Table 1) and proliferating $\left(\mathrm{Ki} 67^{+}\right)$epidermal cell numbers were, in general, progressive throughout the study period. Significant epidermal thinning was observed as early as day 8 in the $50 \mathrm{mg} / \mathrm{kg}$ dose group (25\% reduction, $P=0.04)$, with incremental changes observed at day 78 (61\% reduction, $P=0.009)$. The most marked changes in the proliferating epidermal cell numbers were also observed at day 78 in the top 2 dosing cohorts; mean declines from baseline of $82 \%(P=0.004)$ and $72 \%(P=0.03)$ were observed at the 25 and $50 \mathrm{mg} / \mathrm{kg}$ dose levels, respectively.
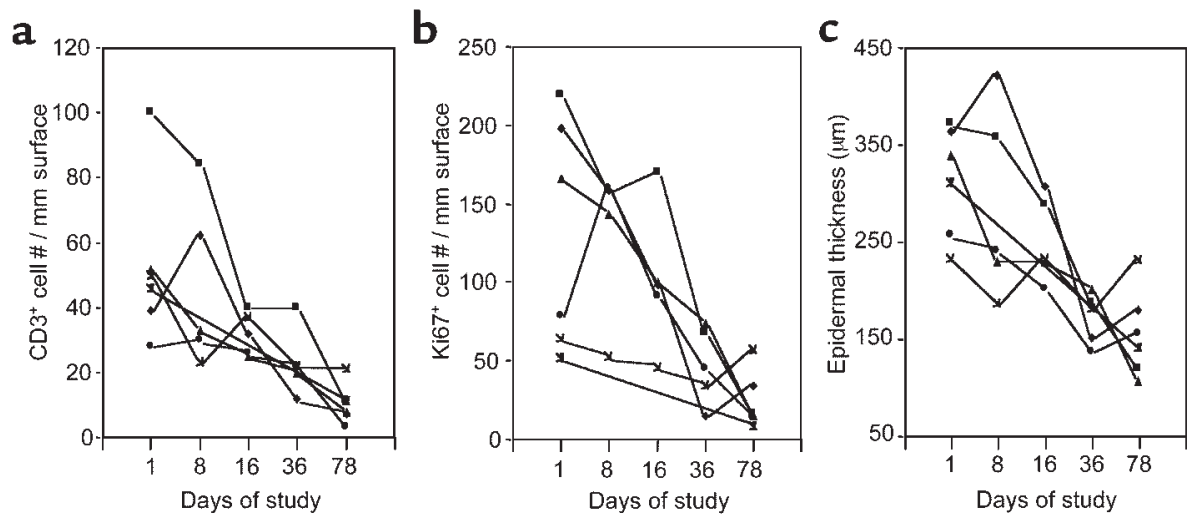

Figure 4

Quantitative histologic parameters measured in the 6 patients accrued to the CTLA4lg $25 \mathrm{mg} / \mathrm{kg}$ dose group. In $\mathbf{a}$ and $\mathbf{b}$, the number of epidermal $\mathrm{CD}^{+}(\mathrm{T})$ cells and the number of $\mathrm{Ki} 67^{+}$(proliferating) keratinocytes per linear millimeter of surface are depicted over the period of observation. In c, the change in epidermal thickness over the first 78 days of the study period is shown. Epidermal thickness was calculated by quantitating the crosssectional epidermal surface area beneath a 1-mm linear region of a representative histologic section using computer-assisted image analysis. Individual data points are an average derived from triplicate analyses. The mean percent changes at day 78 compared with day 1 in a-c were $82 \%(P<$ $0.001), 82 \%(P=0.004)$, and $51 \%(P=0.01)$, respectively. $P$ values were based on a two-sided $t$ test for no change at day 78 compared with day 1 . 
a

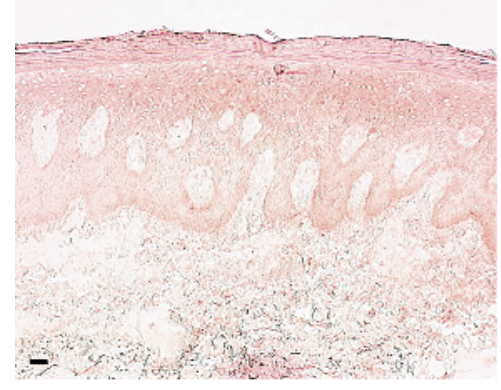

b

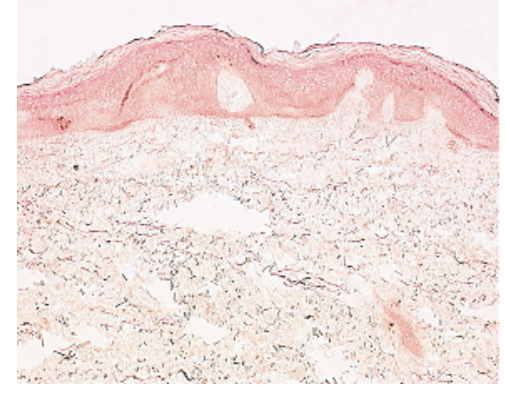

C

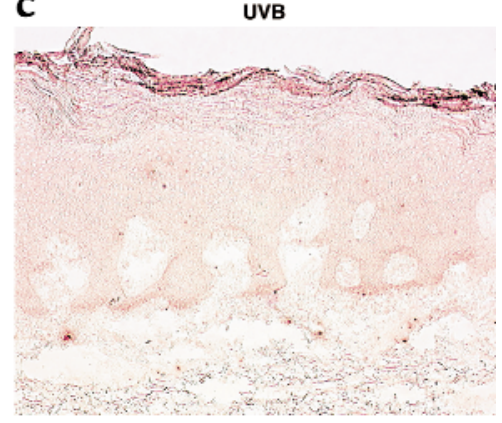

d

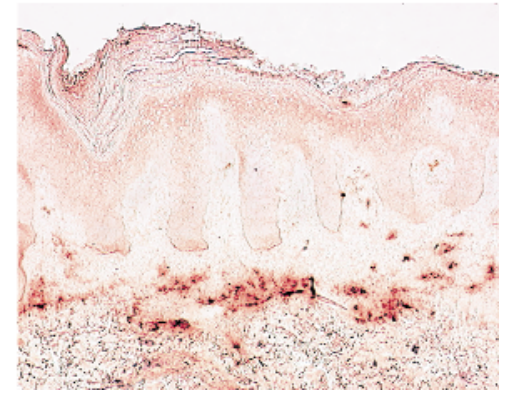

Figure 5

Absence of increased intralesional apoptosis following administration of CTLA4Ig. Illustrated in $\mathbf{a}$ and $\mathbf{b}$ are representative negative TUNEL reactions obtained following histochemical examination across all sampling time points and all dose levels of the study. In representative sections from a patient accrued to the $50 \mathrm{mg} / \mathrm{kg}$ dose level, no increased rate of in situ cell death was evident when comparisons were made between pretreatment (a) and day 36 (b) lesional biopsies. Scale bar in a: $100 \mu \mathrm{m}$. Positive controls included in these experiments are illustrated in $\mathbf{c}$ and $\mathbf{d}$, which are paired biopsy specimens from patients receiving ultraviolet $B$ light (UVB) therapy. An increased rate of in situ apoptosis at day 15 (d) compared with baseline examination (c) was seen following daily administration of UVB treatment. Double staining of these sections with $\mathrm{mAb}$ 's reactive with CD3 identified the apoptotic cells as T cells.
Reductions in epidermal thickness correlated with reductions in infiltrating epidermal T cells $(r=0.60)$. At each dose level, the mean number of epidermal and dermal $\mathrm{T}$ cells decreased serially following day 1 dosing through the final histologic sampling (day 78). Across the 8 dose levels, the most significant decreases in the number of infiltrating epidermal and dermal $\mathrm{T}$ cells compared with baseline were observed at day 78 in the 2 top dosing cohorts. At these 2 highest dose levels, the absolute number of epidermal and dermal intralesional lymphocytes fell to near normal range at day 78 .

One possible mechanism for the diminution of intralesional T-cell numbers is enhanced intralesional T-cell apoptosis. To test this possibility, a TUNEL assay was used to measure apoptosis in skin biopsy material selected across all dose levels and biopsy time points. No increased rate of DNA fragmentation in skin histologic sections was observed following administration of CTLA4Ig (Figure 5). In contrast, apoptotic $\mathrm{T}$ cells were found in lesional biopsies from psoriatic patients receiving ultraviolet B light (UVB), a therapy believed to be associated with in situ Tcell apoptosis (33). These data suggest that the large decrease in intralesional T-cell numbers observed following administration of CTLA4Ig was not attributable to a markedly increased rate of intralesional T-cell apoptosis.

Humoral immune response to bacteriophage $\phi X 174$ and $K L H$. CTLA4Ig has been shown to abrogate a humoral immune response to $\mathrm{T}$ cell-dependent antigens in preclinical models $(27,34,35)$. To test the importance of the CD28/CD152 (CTLA-4) costimulatory pathway in the generation of a $\mathrm{T}$ cell-dependent humoral immune response in humans, study patients were immunized with 2 neoantigens, bacteriophage $\phi X 174$ and KLHImmuneActivator. Suppression of antibody titers to levels 2 or more SDs below the mean of a parallel control group were observed in 1 or more patients accrued to all dose levels following primary immunization with bacteriophage $\phi X 174\left(\phi X 1741^{\circ}\right)$, secondary immunization with bacteriophage $\phi X 174\left(\phi X 1742^{\circ}\right)$, and secondary immunization with keyhole limpet hemocyanin (KLH $2^{\circ}$ ). Suppression of each of these antibody titers was observed most consistently in the 18 patients enrolled in the 8,16 , and $25 \mathrm{mg} / \mathrm{kg}$ dose levels (Table 1). An apparent dose effect was evident in the mean antibody titers measured following $\phi X 1741^{\circ}, \phi X 1742^{\circ}$, and KLH $2^{\circ}$ immunizations in patients receiving CTLA4Ig unit doses of $0.5-25 \mathrm{mg} / \mathrm{kg}$ (Tables 2 and 3). Paradoxically, normal peak antibody titers (predominantly IgM) were observed following bacteriophage $\phi X 174$ and KLH immunizations in several patients in the CTLA4Ig $50 \mathrm{mg} / \mathrm{kg}$ dosing cohort. At unit doses of $1 \mathrm{mg} / \mathrm{kg}$ CTLA4Ig or greater, the percent of anti-bacteriophage antibody of IgG isotype was significantly reduced compared with control values (Table 2). This is consistent with a role for CD28/CD152 in antibody class switching (35), a T cell-dependent event.

Across all doses of CTLA4Ig evaluated, the humoral immune response was documented to be reconstituted in study patients administered tertiary (day 71) and quaternary (day 148) immunizations with bacteriophage $\phi X 174$ (Figure 6). There was no evidence of permanent nonresponsiveness (tolerance induction) to this soluble protein antigen. One study patient at the $50 \mathrm{mg} / \mathrm{kg}$ dose level, though demonstrating sequential amplification of the humoral immune response, did not display full reconstitution to within 2 SDs of the primary and secondary antibacteriophage antibody control values following tertiary and quaternary immunizations, respectively. The CTLA4Ig mean $( \pm \mathrm{SD})$ through serum concentrations at days 78 and 148 were $104 \pm 19.6 \mu \mathrm{g} / \mathrm{mL}$ and $12.4 \pm 12.6 \mu \mathrm{g} / \mathrm{mL}$, respectively, in patients accrued to the $50 \mathrm{mg} / \mathrm{kg}$ dose level.

\section{Discussion}

To our knowledge, this report constitutes the first human experience with an agent designed to simultaneously block T-cell costimulatory signals transduced through the CD28 and CD152 (CTLA-4) receptors. The 

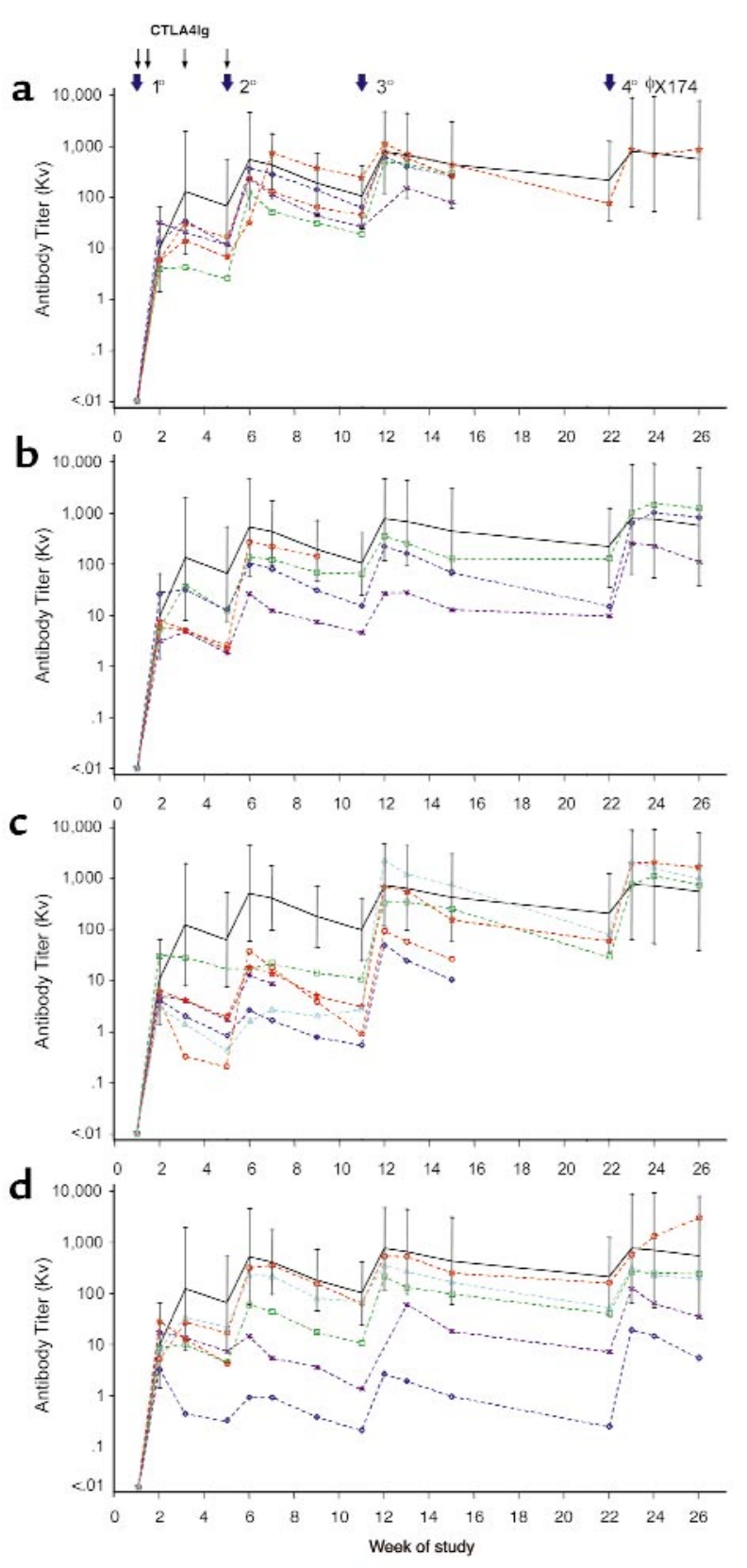

data from this multidose, phase I clinical trial in patients with psoriasis suggest that CTLA4Ig (BMS-188667) may be an effective strategy for immunomodulation of T-cell function and provide insights into the pathogenesis of psoriasis. CTLA4Ig, administered to study patients with stable plaque psoriasis, reduced the number of intralesional $\mathrm{T}$ cells in a dose-dependent manner. The changes in lesional $\mathrm{T}$-cell numbers correlated with reductions in epidermal proliferation, epidermal thickness, reversion of keratinocyte maturational abnormalities, and clinical improvement of psoriasis. The highly selective nature of this therapeutic intervention supports prior findings and suggests that activated $T$ cells, and the cytokines that they elaborate, are the prime effectors in the pathogenesis of psoriasis (2-8).

This study also illustrates the importance of the continued activation of $\mathrm{T}$ cells interacting with $\mathrm{B} 7$ molecules

\section{Figure 6}

Reconstitution of the humoral immune response to bacteriophage $\phi \times 174$ in the $1 \mathrm{mg} / \mathrm{kg}, 4 \mathrm{mg} / \mathrm{kg}, 16 \mathrm{mg} / \mathrm{kg}$, and $50 \mathrm{mg} / \mathrm{kg}$ dosing cohorts following tertiary and quaternary immunization. Bacteriophage $\phi \times 174$ was administered at week $1\left(1^{\circ}\right)$, week $5\left(2^{\circ}\right)$, week $11\left(3^{\circ}\right)$, and week 22 $\left(4^{\circ}\right)$. Patient sera were collected before and 1,2 , and 4 weeks after each administration of bacteriophage. Anti-bacteriophage antibody titers were determined by a neutralizing assay and expressed as a rate of phage inactivation, or $\mathrm{K}$ value (Kv). Antibody titers for patients accrued to the 1 $\mathrm{mg} / \mathrm{kg}(\mathbf{a}), 4 \mathrm{mg} / \mathrm{kg}(\mathbf{b}), 16 \mathrm{mg} / \mathrm{kg}$ (c), and $50 \mathrm{mg} / \mathrm{kg}$ (d) dose groups are depicted. The geometric mean Kv's of the 23 psoriatic control patients are illustrated by the dark black line in each part. Vertical bars indicate $\pm 2 \mathrm{SD}$ of the control group mean Kv. Following tertiary and/or quaternary immunization, all patients' titers were within 2 SD of the control group primary and secondary responses, respectively, with the exception of 1 patient enrolled in the $50 \mathrm{mg} / \mathrm{kg}$ dose group (diamonds in d). Despite progressive titer amplification and a immunoglobulin class switch following the $4^{\circ}$ immunization, this patient did not achieve the study definition of full reconstitution of the humoral immune response to bacteriophage $\phi \times 174$.

on APCs in the maintenance of the psoriatic phenotype. Despite the evidence that signals transduced through CD28 are important in T-cell survival $(36,37)$, no increased rate of T-cell apoptosis was discernible in psoriatic lesions following administration of CTLA4Ig. The observed diminution in lesional T-cell numbers may, therefore, be attributed to factors altering T-cell recruitment into psoriatic lesions $(38,39)$, failure of local expansion of antigen-specific T cells $(40,41)$, and/or apoptosis of antigen-specific $T$ cells at extralesional sites.

The durability of the clinical responses observed following administration of CTLA4Ig was notable in this preliminary clinical experience. At the lower dose levels evaluated, clinical quiescence was maintained well beyond the presence of detectable levels of CTLA4Ig in the serum. The sustained responses in clinical measures of psoriasis activity may be attributed to the degree of normalization of the pathologic epidermal and keratinocyte activation in the psoriatic lesions. Applying similar quantitative methodology, the degree of normalization observed following administration of CTLA4Ig was comparable to psoralen and ultraviolet A light (PUVA) therapy and greater than that observed following cyclosporine treatment $(28,42)$.

In addition to modulation of the cutaneous cell-mediated immune response in patients with psoriasis, CTLA4Ig also demonstrated activity in the humoral limb of the immune system. When comparisons are made between the 2 immunogens employed in this study, peak secondary antibody titers were more suppressed following bacteriophage $\phi X 174$ challenge than after KLH secondary immunization. The immunization schedule of these 2 immunogens varied; both the primary and secondary bacteriophage $\phi X 174$ immunizations occurred following initiation of dosing with CTLA4Ig. Conversely, the KLH immunization schema involved a primary immunization with this immunogen 2 weeks prior to the first dose of CTLA4Ig. The secondary immunization with both immunogens was concurrent with the fourth infusion of CTLA4Ig (day 29). These data, in accordance with murine studies (32), suggest 
that fully primed secondary $\mathrm{T}$ cell-dependent humoral immune responses, such as those elicited to KLH in this study, are less dependent upon T-cell costimulation through the CD28/CD152 pathway $(43,44)$. Therefore, although meaningful clinical responses were noted in this chronic autoimmune disease population characterized by ongoing T-cell activation, lower unit doses of CTLA4Ig may yield equivalent or superior blockade of Tcell activation in clinical settings where primary immune responses dominate.

Paradoxically, normal peak antibody titers following $\mathrm{KLH}$ and bacteriophage $\phi X 174$ immunization were observed in some patients receiving CTLA4Ig unit doses of $50 \mathrm{mg} / \mathrm{kg}$. Though the dose response was fairly consistent across the cell-mediated and humoral biologic end points incorporated in this study, there was clear divergence at this top dose level. A possible explanation could be that at very high unit doses of CTLA4Ig, univalent, lower-affinity binding with B7 molecules (45) would predominate and thereby reduce the effectiveness of CTLA4Ig as a blocking agent. Alternatively, at this top dose level, more efficient blockade of the interaction of B7 with CTLA-4 may ensue, disrupting an inhibitory signal transduced through CTLA-4 (46-49). Further studies are needed to more fully elucidate the mechanisms responsible for these observations.

Tolerance induction has been noted following CTLA4Ig administration in certain preclinical settings (13-15). This immunologic property, however, generally has not been observed following exposure to soluble protein antigens and, similarly, was not evident in this clinical study. Additional studies will be required to explore the optimal schedule of CTLA4Ig administration (50) and combinations with other immunosuppressive agents $(12,16)$ that may lead to antigen-specific nonresponsiveness in clinical settings where tolerance is a desired outcome.

A favorable safety profile was observed in this phase I setting; however, full characterization of the clinical safety of CTLA4Ig awaits larger clinical trials providing increased duration of exposure to this novel therapeutic agent. It is postulated that CD152 (CTLA-4) may be a negative regulator of T-cell activation, and CTLA-4-/mice are known to succumb to a fatal lymphoproliferative disorder at 3 weeks of age (46-48). No such alterations in lymphocyte homeostasis were evident in this study. The combined effect of the simultaneous blockade of CD28 and CD152 receptor-ligand interactions may offer a neutral effect on peripheral T-cell homeostasis. Indeed, the lethal lymphoproliferative disorder associated with CTLA-4 ${ }^{-/-}$mice can be prevented by the administration of CTLA4Ig, presumably because the B7 receptors are blocked from interacting with the intact CD28 ligand $(48,49)$. Furthermore, mice lacking CTLA4, B7-1, and B7-2 do not display a lymphoproliferative disorder (58), additionally supporting the importance of unopposed CD28 signaling for disease progression in CTLA-4 ${ }^{-/-}$mice.

Our results indicate that the maintenance of the psoriatic phenotype is dependent, to a large degree, upon the activation of T cells utilizing the CD28/CD152 costimulatory pathway. This pathway is effectively blocked in a dose-dependent fashion following the administra- tion of CTLA4Ig (BMS-188667). This therapeutic strategy may have great utility in the treatment of other $\mathrm{T}$ cell-mediated diseases and warrants further exploration in additional clinical studies.

\section{Acknowledgements}

The authors wish to thank Robert Bruschini (Bristol-Myers Squibb, Princeton, New Jersey, USA) for help in preparing the figures.

1. Greaves, M.W., and Weinstein, G.D. 1995. Treatment of psoriasis. N. Engl. J. Med. 332:581-588.

2. Demidem, A., Taylor, R., Grammer, S.F., and Streilein, J.W. 1991. T-lymphocyte-activating properties of epidermal antigen-presenting cells from normal and psoriatic skin: evidence that psoriatic epidermal antigenpresenting cells resemble cultured normal Langerhans cells. J. Invest. Dermatol. 97:454-460.

3. Chang, E.Y., et al. 1992. T-cell activation is potentiated by cytokines released by lesional psoriatic, but not normal epidermis. Arch. Dermatol. 128:1479-1485

4. Strange, P., et al. 1993. T-lymphocyte clones initiated from lesional psoriatic skin release growth factors that induce keratinocyte proliferation. J. Invest. Dermatol. 101:695-700.

5. Bata-Csorgo, Z., Hammerberg, C., Voorhees, J.J., and Cooper, K.D. 1995. Kinetics and regulation of human keratinocyte stem cell growth in short-term primary ex vivo culture. J. Clin. Invest. 95:317-327.

6. Bachelez, H., et al. 1998. Treatment of recalcitrant plaque psoriasis with a humanized non-depleting antibody to CD4. J. Autoimmun. 11:53-62.

7. Gottlieb, S.L., et al. 1995. Response of psoriasis to a lymphocyte-selective toxin $\left(\mathrm{DAB}_{389} \mathrm{IL}-2\right)$ suggests a primary immune, but not keratinocyte, pathogenic basis. Nat. Med. 1:442-447.

8. Mueller, W., and Herrman, B. 1979. Cyclosporin A for psoriasis. N. Engl. J. Med. 301:555.

9. Mueller, D.L., Jenkins, M.K., and Schwartz, R.H. 1989. Clonal expansion versus functional inactivation: a costimulatory signaling pathway determines the outcome of T cell antigen receptor occupancy. Annu. Rev. Immunol. 7:445-480.

10. Linsley, P.S., et al. 1991. Binding of the B cell activation antigen B7 to CD28 costimulates T cell proliferation and interleukin 2 mRNA accumulation. J. Exp. Med. 173:721-730.

11. Linsley, P.S., et al. 1991. CTLA-4 is a second receptor for the B cell activation antigen B7. J. Exp. Med. 174:561-569.

12. Sayegh, M.H., and Turka, L.A. 1998. The role of T-cell costimulatory activation pathways in transplant rejection. N. Engl. J. Med. 338:1813-1821.

13. Lenschow, D.J., et al. 1992. Long-term survival of xenogeneic pancreatic islet grafts induced by CTLA4Ig. Science. 257:789-792.

14. Lin, H., et al. 1993. Long-term acceptance of major histocompatability complex mismatched cardiac allografts induced by CTLA4Ig plus donorspecific transfusion. J. Exp. Med. 178:1801-1806.

15. Sayegh, M.H., et al. 1995. CD28-B7 blockade after alloantigenic challenge in vivo inhibits Th1 cytokines but spares Th2. J. Exp. Med. 181:1869-1874.

16. Kirk, A.D., et al. 1997. CTLA4-Ig and anti-CD40 ligand prevent renal allograft rejection in primates. Proc. Natl. Acad. Sci. USA. 94:8789-8794.

17. Reiser, H., and Stadecker, M.D. 1996. Costimulatory B7 molecules in the pathogenesis of infectious and autoimmune diseases. N. Engl. J. Med. 335:1369-1377.

18. Finck, B.K., Linsley, P.S., and Wofsy, D. 1994. Treatment of murine lupus with CTLA4Ig. Science. 265:1225-1227.

19. Cross, A.H., et al. 1995. Long-term inhibition of murine experimental autoimmune encephalomyelitis using CTLA-4-Fc supports a key role for CD28 costimulation. J. Clin. Invest. 95:2783-2789.

20. Webb, L.M.C., Walmsley, M.J., and Feldmann, M. 1996. Prevention and amelioration of collagen-induced arthritis by blockade of the CD28 costimulatory pathway: requirement for both B7-1 and B7-2. Eur. J. Immunol. 26:2320-2328.

21. Larsen, C.P., et al. 1994. Regulation of immunostimulatory function and costimulatory molecule (B7-1 and B7-2) expression on murine dendritic cells. J. Immunol. 152:5208-5219.

22. Symington, F.W., Brady, W., and Linsley, P.S. 1993. Expression and function of B7 on human epidermal Langerhans cells. J. Immunol. 150:1286-1295.

23. Nestle, F.O., Turka, L.A., and Nickoloff, B.J. 1994. Characterization of dermal dendritic cells in psoriasis. J. Clin. Invest. 94:202-209.

24. Jones, E.L., Epinette, W.W., Hackney, V.C., Menendez, L., and Frost, P. 1975. Treatment of psoriasis with oral mycophenolic acid. J. Invest. Dermatol. 65:537-542.

25. Srinivas, N.R., et al. 1996. Pharmacokinetics and pharmacodynamics of CTLA4Ig (BMS-188667), a novel immunosuppressive agent, in monkeys following multiple doses. J. Pharm. Sci. 85:1-4. 
26. Ochs, H.D., Davis, S.D., and Wedgwood, R.J. 1971. Immunologic responses to bacteriophage $\phi X 174$ in immunodeficiency diseases. J. Clin. Invest. 50:2559-2568.

27. Linsley, P.S., et al. 1992. Immunosuppression in vivo by a soluble form of the CTLA-4 T cell activation molecule. Science. 257:792-795.

28. Vallat, V.P., et al. 1994. PUVA bath therapy strongly suppresses immunological and epidermal activation in psoriasis: a possible cellular basis for remittive therapy. J. Exp. Med. 180:283-296.

29. Gavrieli, Y., Sherman, Y., and Ben-Sasson, S.A. 1992. Identification of programmed cell death in situ via specific labeling of nuclear DNA fragmentation. J. Cell. Biol. 119:493-501.

30. Agresti, A., Mehta, C.R., and Patel, N.R. 1990. Exact inference for contingency tables with ordered categories. J. Am. Stat. Assoc. 85:453-458.

31. Hotchin, N.A., Gandarillas, A., and Watt, F.M. 1995. Regulation of cell surface $\beta 1$ integrin levels during keratinocyte terminal differentiation. J. Cell Biol. 128:1209-1219.

32. Gerdes, J., et al. 1984. Cell cycle analysis of a cell proliferation-associated human nuclear antigen defined by the monoclonal antibody Ki-67. J. Immunol. 133:1710-1715.

33. Ozawa, M., et al. 1999. 312-nanometer ultraviolet B light (narrow-band UVB) induces apoptosis of T cells within psoriatic lesions. J. Exp. Med. 189:711-718.

34. Cabrian, K.M., et al. 1996. Suppression of T-cell-dependent immune responses in monkeys by CTLA4Ig. Transplant. Proc. 28:3261-3262.

35. Ronchese, F., Hausmann, B., Hubele, S., and Lane, P. 1994. Mice transgenic for a soluble form of murine CTLA- 4 show enhanced expansion of antigen-specific $\mathrm{CD} 4{ }^{+} \mathrm{T}$ cells and defective antibody production in vivo. J. Exp. Med. 179:809-817.

36. Boise, L.H, et al. 1995. CD28 costimulation can promote T cell survival by enhancing the expression of Bcl- $\mathrm{x}_{\mathrm{L}}$. Immunity. 3:87-98.

37. Van Parijs, L., Ibraghimov, A., and Abbas, A.K. 1996. The roles of costimulation and FAS in T cell apoptosis and peripheral tolerance. Immunity. 4:321-328.

38. Austrup, F., et al. 1997. P- and E-selectin mediate recruitment of T-helper-
1 but not T-helper-2 cells into inflamed tissues. Nature. 385:81-83.

39. Chin, Y.-H., Falanga, V., Taylor, J.R., Cai, J.-P., and Bax, J. 1990. Adherence of human helper/memory T-cell subsets to psoriatic dermal endothelium. J. Invest. Dermatol. 94:413-417.

40. Dunn, D., et al. 1993. T cell receptor V $\beta$ expression in normal human skin. Proc. Natl. Acad. Sci. USA. 90:1267-1271.

41. Chang, J.C.C., et al. 1994. CD8 ${ }^{+} \mathrm{T}$ cells in psoriatic lesions preferentially use T-cell receptor V $\beta 3$ and/or V $\beta 13.1$ genes. Proc. Natl. Acad. Sci. USA. 91:9282-9286.

42. Gottlieb, A.B., et al. 1992. Studies of the effect of cyclosporine in psoriasis in vivo: combined effects on activated $T$ lymphocytes and epidermal regenerative maturation. J. Invest. Dermatol. 98:302-309.

43. Viola, A., and Lanzavecchia, A. 1996. T cell activation determined by T cell receptor number and tunable thresholds. Science. 273:104-106.

44. Iezzi, G., Karjalainen, K., and Lanzavecchia, A. 1998. The duration of antigenic stimulation determines the fate of naive and effector $\mathrm{T}$ cells. Immunity. 8:89-95.

45. Greene, J.L., et al. 1996. Covalent dimerization of CD28/CTLA-4 and oligomerization of CD80/CD86 regulate T cell costimulatory interactions. J. Biol. Chem. 271:26762-26771.

46. Krummel, M.F., and Allison, J.P. 1996. CTLA-4 engagement inhibits IL2 accumulation and cell cycle progression upon activation of resting $\mathrm{T}$ cells. J. Exp. Med. 183:2533-2540.

47. Tivol, E.A., et al. 1995. Loss of CTLA-4 leads to massive lymphoproliferation and fatal multiorgan tissue destruction, revealing a critical negative regulatory role of CTLA-4. Immunity. 3:541-547.

48. Tivol, E.A., et al. 1997. CTLA4Ig prevents lymphoproliferation and fatal multiorgan tissue destruction in CTLA-4-deficient mice. J. Immunol. 158:5091-5094.

49. Mandelbrot, D.A., McAdam, A.J., and Sharpe, A.H. 1999. B7-1 or B7-2 is required to produce the lymphoproliferative phenotype in mice lacking cytotoxic T lymphocyte-associated antigen 4 (CTLA-4). J. Exp. Med. 189:435-440.

50. Perez, V.L., et al. 1997. Induction of peripheral T cell tolerance in vivo requires CTLA-4 engagement. Immunity. 6:411-417. 